\title{
Topologically Robust Transport of Photons in a Synthetic Gauge Field
}

\author{
S. Mittal, ${ }^{1,2}$ J. Fan, ${ }^{1}$ S. Faez, ${ }^{3}$ A. Migdall, ${ }^{1}$ J. M. Taylor, ${ }^{1}$ and M. Hafezi ${ }^{1,2, *}$ \\ ${ }^{1}$ Joint Quantum Institute, NIST/University of Maryland, College Park, Maryland 20742, USA \\ ${ }^{2}$ Department of Electrical and Computer Engineering, University of Maryland, College Park, Maryland 20742, USA \\ ${ }^{3}$ Huygens-Kamerlingh Onnes Laboratorium, Universiteit Leiden, Postbus 9504, 2300 RA Leiden, The Netherlands
}

(Received 2 May 2014; published 20 August 2014)

\begin{abstract}
Electronic transport is localized in low-dimensional disordered media. The addition of gauge fields to disordered media leads to fundamental changes in the transport properties. We implement a synthetic gauge field for photons using silicon-on-insulator technology. By determining the distribution of transport properties, we confirm that waves are localized in the bulk and localization is suppressed in edge states. Our system provides a new platform for investigating the transport properties of photons in the presence of synthetic gauge fields.
\end{abstract}

DOI: 10.1103/PhysRevLett.113.087403

PACS numbers: 78.67.Pt, 42.79.-e, 71.23.Ft

Photons provide a natural and convenient medium to investigate fundamental quantum transport properties $[1,2]$. Using photons, one can selectively excite states, and observe both the spectral and spatial responses throughout the material, which are challenging tasks in electronic systems. Experimental efforts studying gauge fields with photons have been limited to the microwave domain [3-7], while investigations in the optical domain have remained elusive. This is due to the fact that magneto-optic effectsthe simplest source of coupling between gauge fields and photons-are extremely weak at optical frequencies. Recently though, there have been a significant number of proposals to synthesize gauge fields for optical photons [8-13]. In particular, two concurrent experiments showed exemplary signatures of topological edge states through direct imaging $[14,15]$. Here we report the first observation of the robust nature of topologically protected edge states using an analysis of the statistics of transport properties (transmission and delay). We use a 2D lattice of coupled ring resonators with a synthetic magnetic field, implemented using silicon-on-insulator technology. By considering the distribution of Wigner delay times [16,17], we can unambiguously distinguish nonlocalized diffusive transport in lossy edge states from tunneling through localized bulk states. Finally, we compare the transmission of topologically ordered edge states to the transmission in a topologically trivial one-dimensional system.

Our experiments are performed on a two dimensional lattice of coupled ring resonators [18] [Fig. 1(a)]. The ring resonators are coupled using another set of link rings which are designed to be antiresonant to the main ring resonators, i.e., the length of the connecting rings is slightly longer than the main rings so as to acquire an extra $\pi$ phase shift. The link resonators are spatially shifted, along the $y$ axis, with respect to the main lattice-site resonators such that transiting photons acquire a phase $y \phi$ when hopping along the $x$ axis at a lattice site with row index $y$ [8]. Therefore, a round trip along any plaquette [consisting of 4 ring and 4 link resonators, see Fig. 1(a)] results in a total accumulated phase of magnitude $\phi$ with a \pm sign corresponding to the direction (clockwise or counterclockwise) of travel along the plaquette. Here we only excite and measure the counterclockwise mode in the main ring resonators, with the input port as indicated in Fig. 1(a). This system is equivalent to a uniform synthetic magnetic field with flux $\phi$ penetrating each plaquette of a $2 \mathrm{D}$ photon gas, with the tight-binding Hamiltonian

$$
\begin{aligned}
H_{0}= & -J \sum_{x, y} \hat{a}_{x+1, y}^{\dagger} \hat{a}_{x, y} e^{-i \phi y}+\hat{a}_{x, y}^{\dagger} \hat{a}_{x+1, y} e^{i \phi y} \\
& +\hat{a}_{x, y+1}^{\dagger} \hat{a}_{x, y}+\hat{a}_{x, y}^{\dagger} \hat{a}_{x, y+1},
\end{aligned}
$$

where $J$ is the coupling rate between the on-site rings, and $\hat{a}_{x, y}$ and $\hat{a}_{x, y}^{\dagger}$ are the photon annihilation and creation operators at a main resonator site with indices $x, y$.

For an infinite lattice, the energy eigenvalues of this Hamiltonian constitute the famous Hofstadter butterfly spectrum [8]. The eigenvalues group into allowed energy bands separated by band gaps, forming a topological insulator. For a finite lattice, the band gaps are populated with so-called edge states. The edge states are unidirectional, clockwise (or counterclockwise) propagating states, with their wave function confined to the perimeter of the lattice. We call these long-edge and short-edge states [Fig. 1(a)], respectively, because of the length they travel along the lattice edge from input to output port. These states are in sharp contrast with the eigenstates in the allowed energy bands, which are called bulk states. In the presence of lattice disorder, such as resonance frequency mismatch and coupling variations, bulk states become localized as the sample size exceeds the localization length [Figs. 1(c)-(f)] [19]. In our system, bulk states are localized in even the smallest samples $(4 \times 4)$. Edge states, on the other hand, are topologically protected and their wave functions are robust 

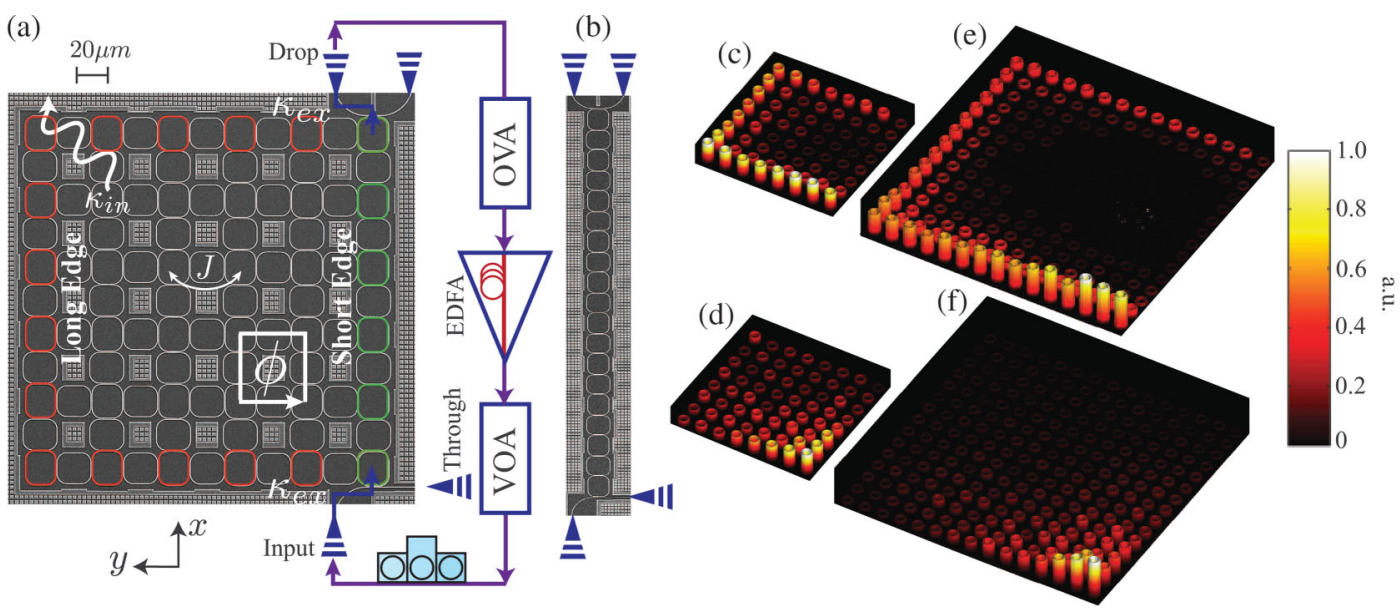

FIG. 1 (color). (a) SEM image of a 2D lattice with the measurement setup. Light is coupled into the lattice or chain at input port exciting a counterclockwise rotating mode. The output at the drop port is measured using an optical vector network analyzer (OVA). An erbium-doped fiber amplifier (EDFA) and variable optical attenuator (VOA) are used to control input power along with a polarization controller. (b) SEM image of a 1D device with 10 main rings. Main rings are coupled using link rings similar to 2D devices. (c) Simulated edge state intensity and (d) bulk states intensity for an $8 \times 8$ lattice averaged over 50 realizations. The edge states span the long edge of the lattice, whereas the bulk states are localized near the input port. (e),(f) Simulated intensity images for edge and bulk states in a $15 \times 15$ lattice. The long-edge wave function still extends across the lattice edge, but the bulk state is again localized with localization extent independent of lattice size.

against disorder in the lattice. As a result, edge state wave functions propagate along the entire edge of the lattice irrespective of the lattice size, although their intensity falls due to absorption and scattering loss from waveguide surface roughness. Our goal here is to leverage quantitative measurements of transmission and delay time to unambiguously demonstrate the robust nature of edge states and distinguish them from bulk states. We show that edge state transport is diffusive and the delay distribution is Gaussian and centered at the average, while for bulk states the delay distribution is asymmetric with the peak value being well below the average, similar to the localization typical in one dimension [17,20,21].

We implemented this system using silicon-on-insulator (SOI) technology. The devices were fabricated at the IMEC foundry using deep-UV projection photolithography [14]. The resonator waveguide cross section is $510 \mathrm{~nm}$ in width and $220 \mathrm{~nm}$ in height, which allows only a single TE mode to exist in waveguides. The coupling region between all rings consists of a linear waveguide section of $7 \mu \mathrm{m}$ with coupling gap of $180 \mathrm{~nm}$, resulting in a uniform coupling rate $J$. The system is probed using input and output waveguides coupled to the lattice, with a coupling rate $\kappa_{\text {ex }}$ [Fig. 1(a)]. Light coupled to the lattice at the input port travels through the lattice and appears at the drop port. The fraction of input light which does not couple to the lattice travels to the through port. The light backscattered, due to waveguide surface roughness and reflections in the coupling region, is directed to the backscattering port. The backscattered light intensity is about $30 \mathrm{~dB}$ lower than that observed at the drop port, indicating negligible backscattering. The main disorder terms affecting our system are the resonance frequency mismatch of the main ring resonators $\left(\Delta \omega_{0}\right)$, variations in the resonator coupling strength $(\Delta J)$, variations in the resonator loss rate $\left(\Delta \kappa_{\text {in }}\right)$ and disorder in the hopping phase $(\Delta \phi)$. We have characterized all these disorder contributions to our system using single ring devices [22]. For transmission and delay-time measurements, we use an optical vector analyzer (LUNA OVA5000) based on swept wavelength interferometry [25]. The Wigner delay time (single channel) for propagation is then calculated as a derivative of phase with respect to angular frequency. Unlike transport time, Wigner time can be negative for anomalous dispersion regions around a phase jump. The negative delay values also appear in simulations where the delay is calculated similarly.

Figures 2(a) and 2(b) show the observed transmission and delay spectra at the drop port for eight different $8 \times 8$ lattice size devices. While the spectra differ significantly because of intrinsic fabrication variations in waveguide dimensions, we can already see the first manifestation of robust edge states in the form of two regions with suppressed variance across devices, in both the transmission and delay spectra (red and green shaded). Since edges states are topologically constrained to travel along the lattice edge, device-to-device fabrication variations in system parameters do not affect the edge state wave functions as much as they do for bulk states. Edge states therefore show reduced variation. Using numerical modeling including our measured values for disorder [22], as shown in Figs. 2(c) and 2(d), we can identify these regions as the long edge and the short edge.

We next analyze the delay distribution to distinguish the behavior of edge and bulk states. This approach provides an 
(a)

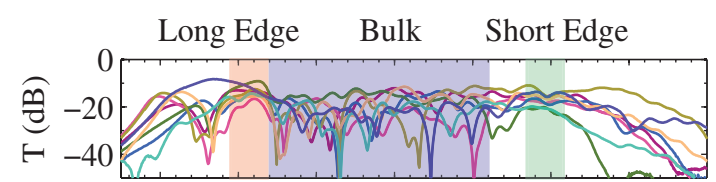

(b)

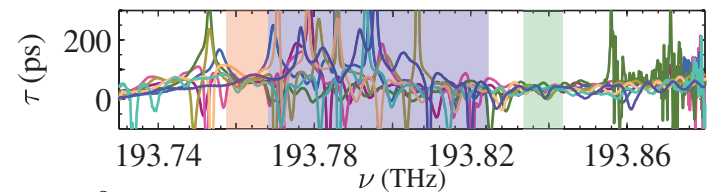

(c)

(d)

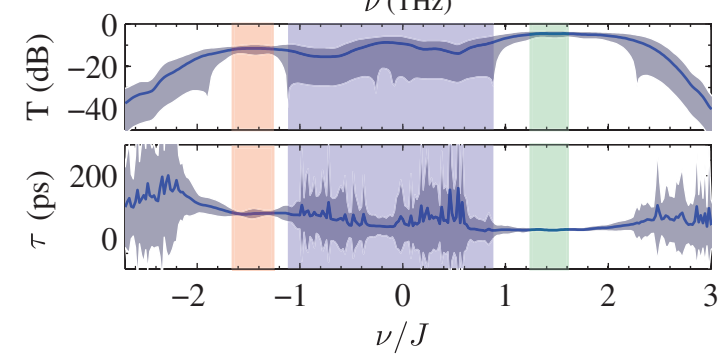

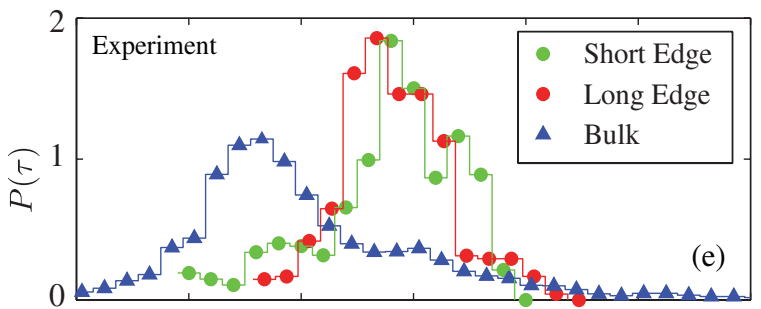

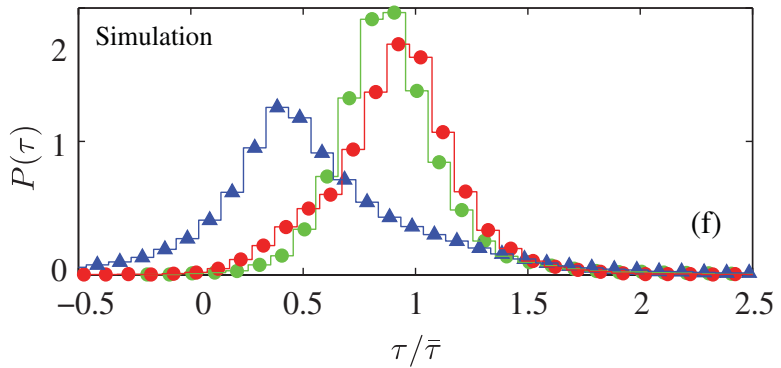

FIG. 2 (color). (a) Measured transmission and (b) delay-time spectra for eight $8 \times 8$ lattice size devices. The spectra have been normalized and shifted along the $x$ axis to superpose them [22]. Two regions with reduced variance in transmission and delay are indicated (shaded red and green). The noisy bulk states region is shown in blue. (c),(d) Simulated transmission and delay with the average (solid blue line) and 95\% confidence band (gray shaded area) determined from the standard deviation across devices. (e),(f) Measured and simulated delay statistics for edge and bulk states. The delay distribution for edge states is Gaussian, indicating diffusive transport. For bulk states the distribution is asymmetric, showing localized transport. Data is taken across 8 devices. The delays are normalized to the average (rms) and the overall delay distribution is normalized to the in-band average and the delay distribution is normalized such that the area under the curve is unity.

unequivocal signature of localization [17,20,26]. Figures 2(e) and 2(f) show the measured and simulated delay distributions for the edge and bulk states in $8 \times 8$ lattice-sized devices and highlight the remarkable difference between edge and bulk states. For edge states, the delay distribution normalized to its average (in actuality, we used root mean square to allow for negative delay values) is approximately Gaussian with a Gaussian width independent of system size. This behavior is characteristic of diffusive transport as seen previously in one-dimensional systems [18]. The bulk state distribution is, however, asymmetric with the most probable value being less than the average. This feature is reminiscent of transport governed by localization which also has been observed earlier in the microwave regime for one-dimensional systems [17]. For localized transport, the delay spectrum exhibits spikes [see Fig. 2(b)] which manifest in the asymmetric delay statistics. These spikes appear due to resonant tunneling through localized states in the bulk [27]. Therefore, even in the presence of loss, delay distribution can clearly differentiate two different regimes of transport in the same photonic system. Our measured results show a good match with numerical modeling. We observe similar behavior for other lattice sizes as well [22].

A test to further establish the topologically protected nature of edge states would be a comparison of the transmission scaling with system size for an edge state against that of a topologically trivial 1D system [18,28], both with similar degrees of disorder. Fabrication-induced disorder in a $1 \mathrm{D}$ ring resonator array leads to a spread in the resonance wavelengths of the resonators. This impedes the forward propagation of light, increases backreflection, i.e., less light is coupled into the array, and, hence, the transmission at output is reduced [29]. Ultimately, as the array length increases, Anderson localization halts transmission of the light [30-32]. Edge states, on the other hand, are unidirectional and immune to reflection caused by disorder. Therefore, transmission through edge states is expected to be less affected.

Figure 3(a) shows the measured average transmission and its standard deviation across a number of chips (95 in total) for the long-edge state band in 2D lattice and the midband of the 1D array as a function of system size, i.e., the number of resonators traveled from input to output (excluding the link resonators). Transmission in both the long-edge state as well as the 1D system decays exponentially with system size. A linear fit to measured transmission (in $\mathrm{dB}$ ) in the long-edge band gives the decay slope as $-0.75(20) \mathrm{dB}$ per ring but for $1 \mathrm{D}$ transmission the slope is $-0.93(16) \mathrm{dB}$ per ring, where uncertainties represent one sigma standard deviation. Transmission along the longedge state can be seen to decay at a slower rate when compared to 1D transport. Simulation results using the experimentally estimated parameters are also presented in the figure. The simulated transmission decay slopes are $-0.66(2)$ and $-1.06(5) \mathrm{dB}$ per ring, respectively, for the 

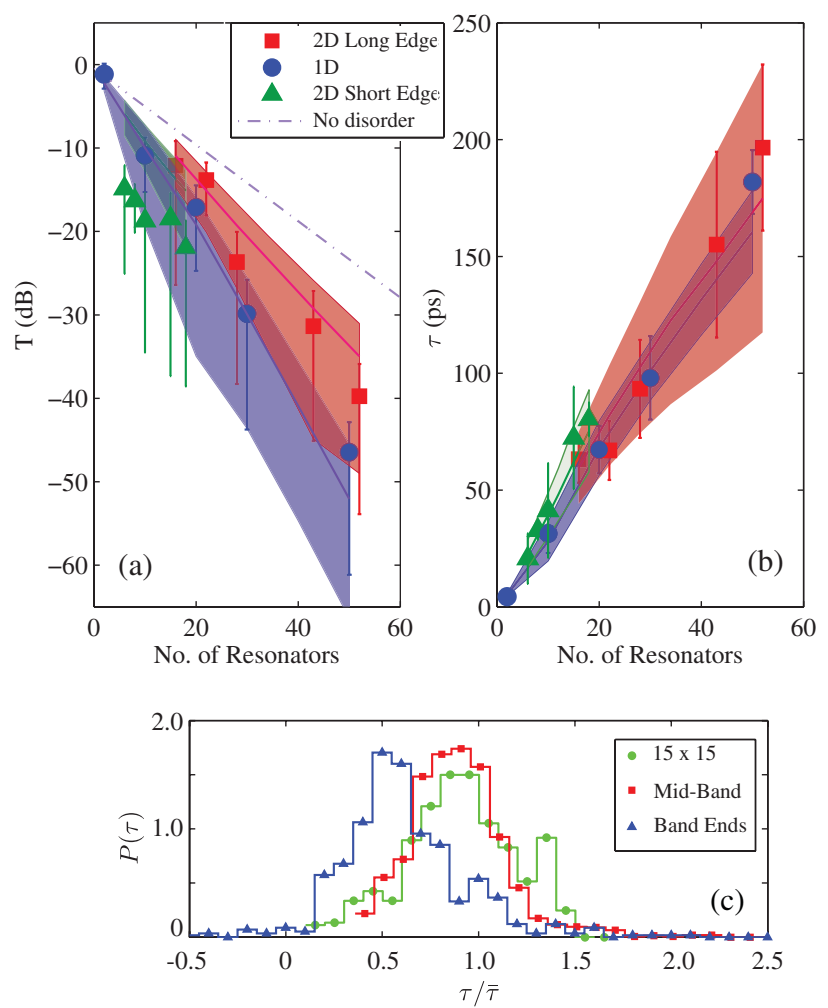

FIG. 3 (color). (a) Transmission and (b) delay-time scaling for 2D and 1D devices. Solid markers with error bars are the measured average and standard deviation (65\% confidence band) values. Solid lines with shaded areas are the simulated average and standard deviation. Also shown is the transmission when there is no disorder in the system. For $2 \mathrm{D}$ data, we measured $(7,8,9,8,8)$ the number of chips for $(6 \times 6,8 \times 8,10 \times 10$, $15 \times 15,18 \times 18 \mathrm{ring})$ sized devices, respectively. For the $1 \mathrm{D}$ data, we measured $(11,15,11,12,6)$ number of chips for $(2,10,20,30,50)$ ring devices. (c) Delay statistics for the long edge of $15 \times 15$ lattice sized devices, midband, and band ends of 30-ring 1D devices. The long edge and midband of 1D devices show diffusive transport. The band ends in 1D devices, however, show localization.

long-edge state and one dimension. The experimental and simulation results are seen to agree, given that the number of devices measured for each lattice size is only $\approx 8$ versus the 5000 realizations for each simulation. In order to differentiate the decay of transmission with system size resulting from resonator losses characterized by $\kappa_{\text {in }}$, from losses due to disorder-both resulting in exponential attenuation-we plot the simulated result for transmission without disorder (presented as a dashed line). In that zero disorder limit, both the 2D and 1D systems are similarly attenuated by loss with a decay rate of $0.46 \mathrm{~dB}$ per ring. We, therefore, observe that disorder affects both $2 \mathrm{D}$ and $1 \mathrm{D}$ systems, but transport in edge states is less susceptible to disorder.

Figure 3(b) shows the measured and simulated average delay and its standard deviation for short-edge and long-edge-state bands. The measured delay, when plotted against the number of rings on the short and long edges of the lattice, increases linearly with a slope 3.9(9) ps and 5.4(1.0) ps per ring respectively for longand short-edge states. The simulated delay slopes are 3.2(2) and 4.4(1) ps per ring, respectively. Again, the experimental results are in agreement with simulations. Also shown in the figure, for comparison, is the measured delay in 1D devices. That delay follows the same scaling as the edge states. However, it can be seen that the standard deviation in delay for $1 \mathrm{D}$ devices is less than that for edge states. This is contrary to the case of a uniform magnetic field where the standard deviation of delay in edge states remains smaller than in a 1D system [8]. Using simulations we have verified that this is due to the fabrication disorder of $\Delta \phi$ in the 2D lattice. We further compare the delay distribution for edge states and a $1 \mathrm{D}$ system. The normalized delay distribution for long-edge states in a $15 \times 15$ lattice, and for midband and band ends of a 30 ring 1D array are shown in Fig. 3(c). We see that for both edge states and the midband of a 1D array, transport is diffusive, the distribution is Gaussian and the width of the distribution is independent of system size. However, the band edges of the 1D array are localized. Using simulations, we also find that as the array length increases beyond 70 rings, even the midband of the $1 \mathrm{D}$ system shows localization.

The silicon-on-insulator technology provides a suitable platform to investigate the statistical effects of synthetic gauge fields on various transport properties and to demonstrate the localization of bulk states and the robustness of edge states. Such a system could pave the way to investigate the effects of other gauge fields, including those with magnetic monopoles and floquet properties, with or without Abelian features on bosonic transport. Moreover, the addition of enhanced optical nonlinearity in these ring resonator structures opens the door to intriguing questions on the nature of solutions for nonlinear transport in systems with topological order.

Certain commercial equipment, instruments, or materials are identified in this Letter to foster understanding. Such identification does not imply recommendation or endorsement by the National Institute of Standards and Technology, nor does it imply that the materials or equipment are necessarily the best available for the purpose.

We thank A. Lobos, S. Ganeshan, and J. Keutchayan for fruitful discussions. This research was supported by the U.S. Army Research Office Multidisciplinary University Research Initiative Grant No. W911NF0910406, and the NSF through the Physics Frontier Center at the Joint Quantum Institute. S. F. acknowledges financial support from the European Research Council (Advanced Grant SiMoSoMa). 
*Corresponding author. hafezi@umd.edu

[1] M. Segev, Y. Silberberg, and D. N. Christodoulides, Nat. Photonics 7, 197 (2013).

[2] A. Lagendijk, v. B. Tiggelen, and D. Wiersma, Phys. Today 62, 8, 24 (2009).

[3] S. Raghu and F. D. M. Haldane, Phys. Rev. A 78, 033834 (2008).

[4] Z. Wang, Y. Chong, J. D. Joannopoulos, and M. Soljačić, Nature (London) 461, 772 (2009).

[5] R. A. Sepkhanov, A. Ossipov, and C. W. J. Beenakker, Europhys. Lett. 85, 14005 (2009).

[6] S. R. Zandbergen and M. J. A. de Dood, Phys. Rev. Lett. 104, 043903 (2010).

[7] X. Wang, H. T. Jiang, C. Yan, Y. Sun, Y. H. Li, Y. L. Shi, and H. Chen, Europhys. Lett. 103, 17003 (2013).

[8] M. Hafezi, E. A. Demler, M. D. Lukin, and J. M. Taylor, Nat. Phys. 7, 907 (2011).

[9] R. O. Umucalilar and I. Carusotto, Phys. Rev. A 84, 043804 (2011).

[10] K. Fang, Z. Yu, and S. Fan, Nat. Photonics 6, 782 (2012).

[11] Y. E. Kraus, Y. Lahini, Z. Ringel, M. Verbin, and O. Zilberberg, Phys. Rev. Lett. 109, 106402 (2012).

[12] M. C. Rechtsman, J. M. Zeuner, A. Tünnermann, S. Nolte, M. Segev, and A. Szameit, Nat. Photonics 7, 153 (2013).

[13] A. B. Khanikaev, S. H. Mousavi, W.-K. Tse, M. Kargarian, A. H. MacDonald, and G. Shvets, Nat. Mater. 12, 233 (2013).

[14] M. Hafezi, S. Mittal, J. Fan, A. Migdall, and J. M. Taylor, Nat. Photonics 7, 1001 (2013).

[15] M. C. Rechtsman, J. M. Zeuner, Y. Plotnik, Y. Lumer, D. Podolsky, F. Dreisow, S. Nolte, M. Segev, and A. Szameit, Nature (London) 496, 196 (2013).
[16] A. A. Chabanov, M. Stoytchev, and A. Z. Genack, Nature (London) 404, 850 (2000).

[17] A. A. Chabanov and A.Z. Genack, Phys. Rev. Lett. 87, 233903 (2001).

[18] M. L. Cooper, G. Gupta, M. A. Schneider, W. M. J. Green, S. Assefa, F. Xia, Y. A. Vlasov, and S. Mookherjea, Opt. Express 18, 26505 (2010).

[19] X.-G. Wen, Quantum Field Theory of Many-body Systems (Oxford University Press, New York, 2007).

[20] C. Texier and A. Comtet, Phys. Rev. Lett. 82, 4220 (1999).

[21] F. Xu and J. Wang, Phys. Rev. B 84, 024205 (2011).

[22] See Supplemental Material at http://link.aps.org/ supplemental/10.1103/PhysRevLett.113.087403, which includes Refs. [8,14,17,23,24].

[23] M. L. Cooper, G. Gupta, M. A. Schneider, W. M. J. Green, S. Assefa, F. Xia, D. K. Gifford, and S. Mookherjea, Opt. Lett. 35, 3030 (2010).

[24] B. A. van Tiggelen, P. Sebbah, M. Stoytchev, and A. Z. Genack, Phys. Rev. E 59, 7166 (1999).

[25] D. K. Gifford, B. J. Soller, M. S. Wolfe, and M. E. Froggatt, Appl. Opt. 44, 7282 (2005).

[26] A. Z. Genack, P. Sebbah, M. Stoytchev, and B. A. van Tiggelen, Phys. Rev. Lett. 82, 715 (1999).

[27] K. Y. Bliokh, Y. P. Bliokh, V. Freilikher, S. Savel'ev, and F. Nori, Rev. Mod. Phys. 80, 1201 (2008).

[28] M. Notomi, E. Kuramochi, and T. Tanabe, Nat. Photonics 2, 741 (2008).

[29] C. Ferrari, F. Morichetti, and A. Melloni, J. Opt. Soc. Am. B 26, 858 (2009).

[30] T. Schwartz, G. Bartal, S. Fishman, and M. Segev, Nature (London) 446, 52 (2007).

[31] S. Mookherjea, J. S. Park, S.-H. Yang, and P. R. Bandaru, Nat. Photonics 2, 90 (2008).

[32] J. Topolancik, B. Ilic, and F. Vollmer, Phys. Rev. Lett. 99, 253901 (2007). 